reduces rACC metabolism to normative levels ${ }^{13}$. Thus, mood may be an important factor modulating the ability to engage the neural mechanisms that are associated with optimism bias, such as reduced rACC activity when thinking about negative future events.

Although optimism is usually beneficial, it may not always promote adaptive behavior and can even be detrimental. Optimists may believe they will attain a goal even when they do not possess the ability to effectively pursue that goal ${ }^{14}$ or to respond to setbacks that occur ${ }^{15}$. For instance, optimism is sometimes negatively correlated with academic achievement ${ }^{14}$. Moreover, it can lead to false beliefs that things will turn out well, which may explain why optimism can be associated with risky behavior ${ }^{14}$. Optimism can also lead to disappointment if one's high expectations are not realized. Indeed, there are times when it is maximally adaptive to shift from optimistic to more realistic, or even pessimistic, expectations, such as when goals are important or a negative outcome is probable ${ }^{14}$. In such instances, pessimism can facilitate preparedness and the use of strategies to reduce the occurrence or consequences of negative outcomes.

These limitations on the benefits of optimism make sense from the brain-based perspective suggested by Sharot et al. ${ }^{5}$. If optimism bias reflects a relative reduction of activity in emotional brain regions when we contemplate a negative future outcome, it is not surprising that sometimes our benign outlooks will prove misleading. In light of evidence that people shift to a more pessimistic outlook when a negative outcome is probable, this reduction might not occur when people imagine negative events that are likely to happen in the near future; in Sharot et al. ${ }^{5}$, subjects imagined negative events that were not likely to happen anytime soon.

We agree with a previous study ${ }^{15}$ that concluded on the basis of psychological evidence that, "People should be optimistic enough to take advantage of the many benefits of a positive outlook, but they should also sufficiently temper that optimism so that they can motivate preventative action and avoid being caught off guard." If the brain is often biased towards promoting a rosy vision of the future, a healthy dose of realism is required to keep the picture in balance.

1. Scheier, M.F., Carver, C.S. \& Bridges, M.W J. Pers. Soc. Psychol. 67, 1063-1078 (1994).

2. Nes, L.S. \& Segerstrom, S.C. Pers. Soc. Psychol. Rev. 10, 235-251 (2006).

3. Weinstein, N.D. J. Pers. Soc. Psychol. 39, 806-820 (1980).

4. Gilbert, D.T. \& Wilson T.D. Science 317, 1351-1354 (2007).

5. Sharot, T., Riccardi, A.M., Raio, C.M. \& Phelps, E.A. Nature published online 24 October 2007.

6. Schacter, D.L., Addis, D.R. \& Buckner, R.L. Nat. Rev. Neurosci. 8, 657-661 (2007).

7. Stark, C.E.L. \& Squire, L.R. Proc. Natl. Acad. Sci. USA 98, 12760-12765 (2001).

8. Phelps, E.A. Annu. Rev. Psychol. 57, 27-53 (2006).

9. Kensinger, E.A. \& Schacter, D.L. Neuropsychologia 43 , 1429-1443 (2005)

10. Brown, G.P., Macleod, A.K., Tata, P. \& Goddard, L. Anxiety Stress Coping 15, 1-17 (2002)

11. Williams, J.M.G. et al. Mem. Cognit. 24, 116-125 (1996).

12. Miranda, R. \& Mennin, D.S. Cognit. Ther. Res. $\mathbf{3 1}$ 71-82 (2007).

13. Drevets, W.C. Biol. Psychiatry 48, 813-829 (2000).

14. Shorey, H.S. et al. Psychol. Inq. 13, 322-331 (2002)

15. Sweeny, K., Carroll, P.J. \& Shepperd, J.A. Curr. Dir. Psychol. Sci. 15, 302-306 (2006).

\title{
Olfactory axons derailed in the brain
}

Yao et al. examine how sensory axons influence patterning of olfactory glomeruli in Drosophila melanogaster on page 1423 of this issue. Olfactory sensory neurons send their axons into the antennal lobes of the brain, where they synapse onto dendrites of matching projection neurons in the glomeruli. Olfactory axons also send a collateral branch across the midline into the contralateral lobe. Both sensory and projection neurons are required for proper patterning of the glomeruli.

The authors initially observed that Wnt5 overexpression in olfactory sensory neurons resulted in ectopic glomeruli forming near the midline. In flies lacking Wnt5, the antennal lobes appeared to be shortened. No glomeruli were missing, but dorsomedial glomeruli were displaced ventrally. The sensory axon branches that normally cross the midline were unable to do so and adopted an aberrant dorsal ipsilateral path. As often happens with fly mutants, the phenotype varied substantially from animal to animal. The photo shows a Wnt5-null pupa showing a relatively mild case. The antennae, with green GFP expressed in olfactory sensory neurons, are at the bottom. The olfactory axons in a double-S curve project to the antennal lobes, which appear yellow because of red counterstaining for Bruchpilot, a synaptic protein that labels all neuropil. In this individual, many olfactory

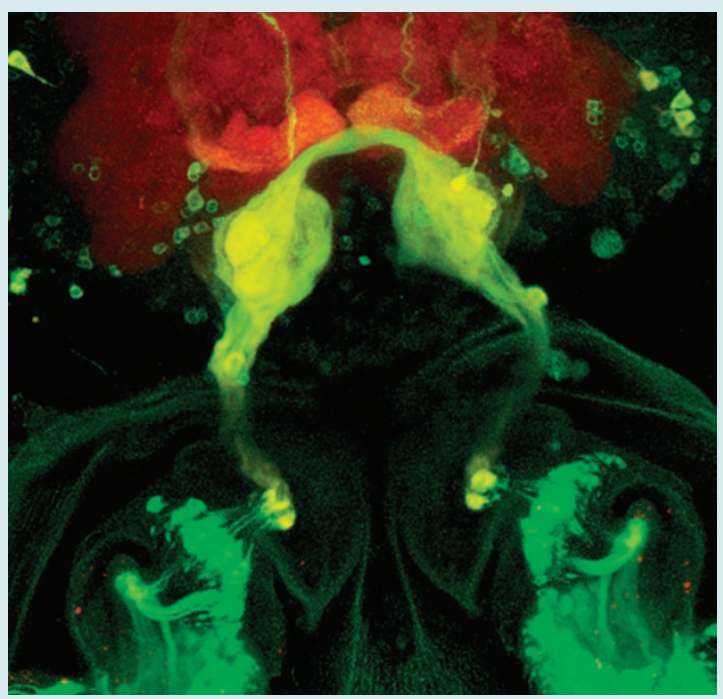
axons do cross the midline, forming a thick commissure between the antennal lobes. Misrouted olfactory axon branches are nevertheless apparent, extending dorsally and ipsilaterally from the antennal lobes straight across the fly brain at the top center.

Wnt5 is a secreted protein that interacts with receptors of the Frizzled and Ryk/Derailed families to exert pleiotropic effects, including axon repulsion, in other systems. The authors show that Wnt5 in olfactory axons is required for proper development of the antennal lobes. How could its absence cause axon misrouting? Yao et al. propose an indirect mechanism, in which Wnt5 secreted from the axons directs crucial aspects of antennal lobe maturation. Looking for the target of axonal Wnt5, the authors report that Derailed (Drl) is strongly expressed in the projection neurons as well as in midline glia. Flies lacking Drl showed a disrupted antennae phenotype that was, in some aspects, such as ectopic glomeruli at the midline, reminiscent of Wnt5 overexpression. Selectively expressing Drl in the null mutant's glia rescued antennal morphology. Furthermore, the intracellular domain of glial Drl was not required for it to fulfill its role in antennal patterning, whereas the extracellular domain was essential. Together with the fact that either too much Wnt5 or a lack of Drl causes the appearance of ectopic glomeruli, these findings suggest that glial Drl antagonizes Wnt5 action at the midline, perhaps by binding Wnt5 and thereby limiting its activation of another, as-yet unidentified receptor. Such midline modulation of Wnt 5 signaling, through further unknown steps, could affect both midline crossing of sensory axon branches and the exclusion of glomeruli from the midline.

Annette Markus 